\title{
TANGENT SPACES TO SCHUBERT VARIETIES
}

\section{LAKSHMIBAI}

In this note, we announce a criterion for smoothness of a Schubert variety in the flag variety $G / B$. Let $G$ be a semi-simple, simply connected algebraic group, which we assume for simplicity to be defined over an algebraically closed field $k$ of characteristic 0 . (The following discussion is valid in any characteristic, in fact even over $\mathbf{Z}$.) Let $T$ be a maximal torus in $G$, and $W$ the Weyl group. Let $R$ be the system of roots of $G$ relative to $T$. Let $B$ be a Borel subgroup of $G$, where $B \supset T$. Let $S$ (resp. $R^{+}$) be the set of simple (resp. positive) roots of $R$ relative to $B$. For $\alpha \in R$, let $s_{\alpha}$ be the reflection. For $\alpha \in R^{+}$, let $E_{\alpha}, F_{\alpha}$ denote the elements of the Chevalley basis for $\mathfrak{g}(=\operatorname{Lie} G)$, corresponding to $\alpha$. For $w \in W$, let $e_{w}$ be the point in $G / B$, and $X(w)(=\overline{B w B}(\bmod B))$, the Schubert variety, associated to $w$. Let $T\left(w, e_{i d}\right)$ be the the tangent space to $X(w)$ at $e_{i d}$. Let

$$
N_{w}=\left\{\beta \in R^{+} \mid F_{\beta} \in T\left(w, e_{i d}\right)\right\} .
$$

Now $T\left(w, e_{i d}\right)$ being a $T$-submodule of the $T$-module $\sum_{\alpha \in R^{-}} \mathfrak{g}_{\alpha}$ (here $R^{-}$ denotes the set of negative roots in $R$, and $\mathfrak{g}_{\alpha}$ denotes the root space $k F_{\alpha}$ ), we have

$$
T\left(w, e_{i d}\right)=\text { the span of }\left\{F_{\beta}, \beta \in N_{w}\right\}
$$

For a dominant weight $\lambda$, let $V(\lambda)$ be the irreducible $G$-module (over $\mathbf{C}$ ) with highest weight $\lambda$. Let us fix a highest weight vector $u$ in $V(\lambda)$. For $w \in W$, let $u_{w}=w \cdot u$, and $V_{w}=U^{+}(\mathfrak{g}) u_{w}$ (here $U^{+}(\mathfrak{g})$ is the subalgebra of $U(\mathfrak{g})$, the universal enveloping algebra of $\mathfrak{g}$, generated by $\left.\left\{E_{\alpha}, \alpha \in S\right\}\right)$. For a weight $\mu$ in $V(\lambda)$, let $m(\mu)$ (resp. $m_{w}(\mu)$ ) denote the multiplicity of $\mu$ in $V(\lambda)\left(\operatorname{resp} . V_{w}\right)$.

Received December 12, 1994; revised April 19, 1995.

Partially supported by NSF Grant DMS 9103129. 
Theorem 1. Let $\beta \in R^{+}$, and $\rho=\frac{1}{2}$ the sum of positive roots. Then $\beta \in N_{w}$ if and only if $m_{w}(\rho-\beta)=m(\rho-\beta)$.

As a consequence, we obtain a criterion for the smoothness of a Schubert variety as given by the following

Corollary. Let $w \in W$, and $M_{w}=\left\{\beta \in R^{+} \mid m_{w}(\rho-\beta)=m(\rho-\beta)\right\}$. Then $X(w)$ is smooth if and only if $\# M_{w}=l(w)$, where $l_{w}$ denotes the length of $w(=\operatorname{dim} X(w))$.

The proof is immediate, since $X(w)$ is smooth if and only if it is smooth at $e_{i d}$.

\section{Outline of a proof of Theorem 1}

For generalities on algebraic groups, one may refer to $[\mathrm{B}]$.

Let us fix a dominant, regular weight $\lambda$. Let $V(\lambda), u, u_{w}, V_{w}$ be as above. We have (cf. [P])

$$
T\left(w, e_{i d}\right)=\text { the span of }\left\{F_{\beta}, \beta \in R^{+} \mid F_{\beta} u \in V_{w}\right\} .
$$

From (2) and (3), we obtain

$$
N_{w}=\left\{\beta \in R^{+} \mid F_{\beta} u \in V_{w}\right\} .
$$

In $[\mathrm{L}]_{3}$, we constructed a basis $\mathcal{B}(\lambda)$ for $V(\lambda)$ which is compatible with the Bruhat order, i.e., $V_{w} \cap \mathcal{B}(\lambda)=\mathcal{B}_{w}(\lambda)$, say, is a basis for $V_{w}$. Further, this basis consists of elements of the form $D u$, where $D$ is either 1 or $F_{\gamma_{1}}^{\left(n_{1}\right)} \cdots F_{\gamma_{r}}^{\left(n_{r}\right)}, \gamma_{i}$ simple, $n_{i}>0$ (for some suitable $n_{i}$ 's), and $s_{\gamma_{r}} \cdots s_{\gamma_{1}}$ is reduced (here $F_{\gamma}^{(n)}=\frac{F_{\gamma}^{n}}{n !}$ ). To be more precise, let

$$
I=\{\text { Lakshmibai-Seshadri paths of shape } \lambda\},
$$

$$
I_{w}=\{\pi \in I \mid w \geq \phi(\pi)\}
$$

notations being as in $[\mathrm{Li}]$. Then it is shown in $[\mathrm{Li}]$,

$$
\operatorname{char} V(\lambda)=\sum_{\pi \in I} e^{\nu(\pi)}
$$

$$
\operatorname{char} V_{w}=\sum_{\pi \in I_{w}} e^{\nu(\pi)}
$$


In particular, using (7), we obtain a formula for $m(\mu), \mu \in X$, the weight lattice, namely

$$
m(\mu)=\#\{\pi \in I \mid \nu(\pi)=\mu\}
$$

Fixing a reduced expression $s_{i_{1}} s_{i_{2}} \cdots s_{i_{r}}$ for $w$, we have (cf. [L] $\left.]_{3}\right)$

$$
\begin{gathered}
I_{w}=\left\{f_{i_{1}}^{n_{1}} \cdots f_{i_{r}}^{n_{r}} \pi_{0}, \text { for suitable } n_{i} \in \mathbf{Z}^{+}\right\}, \\
\mathcal{B}_{w}(\lambda)=\left\{F_{i_{1}}^{\left(n_{1}\right)} \cdots F_{i_{r}}^{\left(n_{r}\right)} u \mid f_{i_{1}}^{n_{1}} \cdots f_{i_{r}}^{n_{r}} \pi_{0} \in I_{w}\right\} .
\end{gathered}
$$

Here, $\pi_{0}$ is the Lakshmibai-Seshadri path given by the line segment in $X \otimes \mathbf{R}$ joining the origin and $\lambda$, and $f_{i}$ are the operators on $I$ as defined in $[\mathrm{Li}])$.

Let us write $\mathcal{B}(\lambda)=\left\{Q_{\pi}, \pi \in I\right\}$. For $\lambda=\rho$, we are able to write down (cf.[L] $]_{4}$ ) very precisely the expression for $F_{\beta} u$ as a linear combination of the elements in $\mathcal{B}(\lambda)$, namely,

$$
F_{\beta} u=\sum_{I^{\rho, \beta}} c_{\pi} Q_{\pi}, \quad c_{\pi} \in k^{*}
$$

where

$$
I^{\rho, \beta}=\{\pi \in I \mid \nu(\pi)=\rho-\beta\}
$$

We have (cf.(9))

$$
m(\rho-\beta)=\# I^{\rho, \beta}
$$

By the Bruhat order compatibility of $\mathcal{B}(\lambda)$, we have (cf.(12))

$$
F_{\beta} u \in V_{w} \Longleftrightarrow Q_{\pi} \in V_{w}, \forall \pi \in I^{\rho, \beta}
$$

Now (14) and (15) imply that

$$
F_{\beta} u \in V_{w} \Longleftrightarrow m_{w}(\rho-\beta)=m(\rho-\beta)
$$

Hence, from (4) and (16), we obtain

$$
\beta \in N_{w} \Longleftrightarrow m_{w}(\rho-\beta)=m(\rho-\beta)
$$




\section{Further consequences}

Given $\beta \in R^{+}$, using the expression for $F_{\beta} u$ as a linear combination of the elements in $\mathcal{B}(\rho)$ as given by (12) above, we are able to describe $N_{w}$ in a very elegant form for classical groups as described in Theorem 2 below. We shall follow the notation in [Bou] to denote the elements of $R^{+}$. We shall denote the Bruhat order in $W$ by $\geq$.

Theorem 2. Let $\beta \in R^{+}$.

(a) Let $G$ be of type $\mathbf{A}_{n}$. Then $\beta \in N_{w} \Longleftrightarrow w \geq s_{\beta}$.

(b) Let $G$ be of type $\mathbf{C}_{n}$.

(1) Let $\beta=\epsilon_{i}-\epsilon_{j}$, or $2 \epsilon_{i}$. Then $\beta \in N_{w} \Longleftrightarrow w \geq s_{\beta}$.

(2) Let $\beta=\epsilon_{i}+\epsilon_{j}$. Then $\beta \in N_{w} \Longleftrightarrow w \geq$ either $s_{\epsilon_{i}+\epsilon_{j}}$ or $s_{2 \epsilon_{i}}$.

(c) Let $G$ be of type $\mathbf{B}_{n}$.

(1) Let $\beta=\epsilon_{i}-\epsilon_{j}, \epsilon_{n}$, or $\epsilon_{i}+\epsilon_{n}$. Then $\beta \in N_{w} \Longleftrightarrow w \geq s_{\beta}$.

(2) Let $\beta=\epsilon_{i}, i<n$. Then $\beta \in N_{w} \Longleftrightarrow w \geq$ either $s_{\epsilon_{i}}$ or $s_{\epsilon_{i}+\epsilon_{n}}$.

(3) Let $\beta=\epsilon_{i}+\epsilon_{j}, j<n$. Then $\beta \in N_{w} \Longleftrightarrow$

$w \geq$ either $s_{\epsilon_{i}+\epsilon_{j}}$ or $s_{\epsilon_{i}} s_{\epsilon_{j}+\epsilon_{n}}$.

(d) Let $G$ be of type $\mathbf{D}_{n}$.

(1) Let $\beta=\epsilon_{k}-\epsilon_{l}$, or $\epsilon_{i}+\epsilon_{j}, j=n-1, n$. Then $\beta \in N_{w} \Longleftrightarrow w \geq s_{\beta}$.

(2) Let $\beta=\epsilon_{i}+\epsilon_{j}, j<n-1$. Then $\beta \in N_{w} \Longleftrightarrow$

$$
w \geq \text { either } s_{\epsilon_{i}+\epsilon_{j}} \text { or } s_{\epsilon_{i}+\epsilon_{n}} s_{\epsilon_{i}-\epsilon_{n}} s_{\epsilon_{j}+\epsilon_{n-1}} \text {. }
$$

Remark 1. The result in Theorem 2 for type $\mathbf{A}_{n}$ is contained in [L-S] also. In $[\mathrm{L}]_{1},[\mathrm{~L}]_{2},[\mathrm{~L}-\mathrm{R}]$, results were obtained towards the determination of $T\left(w, e_{i d}\right)$ for types $\mathbf{C}_{n}, \mathbf{B}_{n}, \mathbf{D}_{n}$, respectively. The formulation of $T\left(w, e_{i d}\right)$ as given in Theorem 2 is a nice refinement of the formulations in loc.cit. Moreover, the method of proof outlined above is much more simple and straightforward than the proofs in loc.cit.

Remark 2. In $[\mathrm{S}]_{2}$ (see also $[\mathrm{S}]_{1}$ ), the authour gives a criterion for smoothness of Schubert varieties in terms of the nil Hecke ring.

\section{Acknowledgement}

The authour expresses her thanks to the referee for important comments and suggestions. 


\section{References}

[B] A. Borel, Linear algebraic groups, W. A. Benjamin, New York, 1969.

[Bou] N. Bourbaki, Groupes et albebres de Lie, Chapitres 4,5, et 6, Hermann, Paris, 1968.

$\left[\mathrm{L}_{1}\right] \quad$ V. Lakshmibai, Geometry of G/P-VII, J. Alg 108 (1987), 403-434.

$\left[\mathrm{L}_{2}\right] \quad$, Geometry of G/P-VIII, J. Alg 108 (1987), 435-471.

$\left[\mathrm{L}_{3}\right]$, Bases for Quantum Demazure modules, to appear in CMS proceedings of the conference on Representations of groups- Finite, Algebraic, Lie, and Quantum, Banff, 1994.

$\left[\mathrm{L}_{4}\right] \longrightarrow$ On tangent spaces to Schubert varieties (in preparation).

[L-R] V. Lakshmibai and K. N. Rajeswari, Geometry of G/P-IX, J. Alg 130 (1990), $122-165$.

[L-S] V. Lakshmibai and C. S. Seshadri, Singular locus of a Schubert variety, Bull. A.M.S. 2 (1984), 363-366.

[Li] P. Littelmann, Littlewood-Richardson rule for symmetrizable Kac-Moody algebras, Inv. Math. 116 (1994), 329-346.

[P] P. Polo, On Zariski tangent spaces of Schubert varieties, and a proof of a conjecture of Deodhar, Indag. Mathem., N.S. 5 (1994), 483-493.

$\left[\mathrm{S}_{1}\right] \quad$ S. Kumar, The nil Hecke ring and singularities of Schubert varieties, Proceedings of the conference in honor of Kostant's 60th birthday, Birkhaüser, 1994.

$\left[\mathrm{S}_{2}\right] \quad$, The nil Hecke ring and singularities of Schubert varieties, preprint, 1995 (detailed version of the above).

M athematics Department, Northeastern Univerity, Boston M A 02115

E-mail address: lakshmibai@neu.edu 